\title{
EL ORIGINALISMO DE INTENCIONES ORIGINALES: TRES TESIS Y DOS OBJECIONES*
}

\author{
LUCIANO LAISE**
}

RESUMEN: en este trabajo me propongo abordar la teoría que se dio en llamar: "originalismo de intenciones originales", es decir, la teoría contemporánea de la interpretación constitucional que mantiene el significado de las normas constitucionales remite a lo que los constituyentes pretendieron significar públicamente. La presente monografía se estructurará con una primera parte en la cual se enunciarán tres aseveraciones sintéticas sobre las directivas interpretativas y los principales presupuestos semánticos de la mencionada teoría originalista. Posteriormente, se efectuará una segunda parte en la cual se esbozarán los lineamientos generales de dos posibles objeciones a la citada teoría de la interpretación constitucional.

PALABRAS CLAVE: Intenciones originales - originalismo - interpretación constitucional - discrecionalidad judicial - regreso al infinito.

\section{ORIGINAL INTENT ORIGINALISM: THREE THESES AND TWO OBJECTIONS}

ABSTRACT: This article is intended to offer an approach to the original intent originalism, which alludes to the contemporary theory of constitutional interpretation, that holds that the meaning of constitutional

\footnotetext{
Este trabajo se enmarca en un proyecto de investigación titulado: "Causas y límites de la discrecionalidad judicial", financiado por la Agencia Nacional de Promoción Científica y Tecnológica (PICT-2013-3199) y también recibió el apoyo de un proyecto de investigación financiado por la Universidad Austral. Versiones previas del presente artículo fueron sometidas a discusión en el I Congreso Iberoamericano de Filosofía del Derecho (Buenos Aires, 2014) y en un seminario organizado por la Universidad Austral (Pilar, 2014). Agradezco al Prof. Manuel García-Mansilla y a la Prof. Pilar Zambrano, ambos pertenecientes a la Facultad de Derecho de la Universidad Austral (Argentina), por haberme brindado muchas ideas y sugerencias que enriquecieron significativamente a una primera versión de este trabajo. También doy gracias a uno de los evaluadores anónimos por haberme sugerido una serie de observaciones que mejoraron la calidad final de este artículo. Todas las imperfecciones remanentes van exclusivamente por mi cuenta.

** Abogado, Universidad de Buenos Aires (Argentina). Becario interno de posgrado nivel II CONICET, con lugar de trabajo en la Facultad de Derecho de la Universidad Austral (ARGENTINA). Correo electrónico: llaise@ius.austral.edu.ar
} 
clauses refers to the intent of the framers. More specifically, this paper is structured in two sections; the first one describes the main interpretative directives and the semantic presuppositions of the original intent originalism, and the second one outlines two objections to the latter theory of constitutional interpretation.

KEYWORDS: Original intent - originalism - constitutional interpretation - judicial discretion - infinite regress

\section{INTRODUCCIÓN}

En los últimos veinticinco años el originalismo se ha vuelto uno de los temas centrales de la teoría constitucional contemporánea en el ámbito estadounidense ${ }^{1}$. Se podría afirmar que los más recientes avances teóricos del originalismo han superado definitivamente las fronteras del ámbito académico en el célebre caso "District of Columbia v. Heller" (2008) de la Corte Suprema de Justicia de los Estados Unidos de Améri$\mathrm{ca}^{2}$. Sin embargo, cabe poner de relieve que el originalismo no configura una aproximación teórica homogénea sino una "familia de teorías" sobre la interpretación constitucional ${ }^{3}$. Con otras palabras, no hay un solo originalismo sino varios.

No obstante, la mayor parte de los autores que defienden alguna variante del originalismo suelen respaldar, implícita o explícitamente, la sistematización de la tradición originalista que ha formulado Lawrence Solum. Este último autor mantiene que existen dos afirmaciones centrales en las cuales convergen todos $-\mathrm{o}$ al menos casi todos- los autores que se autodenominan "originalistas": 1) la tesis de la fijación que mantiene que el contenido semántico de cualquier disposición constitucional fue fijado al momento en que el texto constitucional fue aprobado o ratificado; sal-

1 Solum, Lawrence B. (2011). "What is Originalism?: The Evolution of Contemporary Originalist Theory”, Grant Huscroft and Bradley W. Miller (ed.), en The Challenge of Originalism: Theories of Constitutional Interpretation, New York: Cambridge University Press, pp. $12-41$, p. 12.

2 En junio de 2008, en el caso "Heller V. District of Columbia", la Corte Suprema de los Estados Unidos declaró la inconstitucionalidad de una ley del Distrito de Columbia que prohibía la posesión de armas de fuego en el hogar, bajo el argumento de que esa regulación legal era contraria a la segunda enmienda de la Constitución. En este último caso, el juez Scalia, uno de los máximos referentes del "nuevo originalismo", fue quien lideró el voto de la mayoría. La citada sentencia constituyó uno de los ejemplos paradigmáticos de la aplicación del método interpretativo que propone el "nuevo originalismo". "District of Columbia v. Heller": (2008) 554 U.S. 570.

3 Solum, Lawrence B. (2008). "Semantic Originalism". Illinois Public Law and Legal Theory Research Papers, Series No. 07-24. 176 pp. Disponible en: http://papers.ssrn.com/sol3/papers.cfm?abstract_id=1120244 [fecha de visita: 02 de octubre de 2014] pp. 2-6. 
vo que mediase una reforma o enmienda constitucional. 2) La tesis de la contribución por la cual se afirma que el significado original de la Constitución contribuye al contenido y desarrollo de las prácticas constitucionales actuales ${ }^{4}$. Con todo, es justo reconocer que, incluso en las discusiones internas del originalismo, existen divergencias sobre las implicancias y consecuencias teóricas que se siguen de ambas tesis ${ }^{5}$.

La diferencia específica que caracteriza al originalismo intencionalista es que el significado de las normas constitucionales remite a las intenciones originales de los constituyentes - framers- o también, en el caso estadunidense, a las intenciones de los ratificadores; vale decir, las personas encargadas de ratificar el texto constitucional en las asambleas o convenciones estaduales. La tesis de la contribución mantiene que el originalismo de intenciones originales es capaz de guiar a los intérpretes constitucionales actuales frente a la resolución de casos reales y concretos $^{6}$. En efecto, Bork, uno de los representantes paradigmáticos del originalismo intencionalista, ha sostenido que interpretar el lenguaje de la Constitución a la luz de las intenciones originales de los constituyentes sería la única manera en que la Constitución puede traducirse en derecho vigente ${ }^{7}$. Aún más, el citado autor estadounidense ha llegado a sostener que ninguna otra metodología interpretativa sería capaz de lograr esto último ${ }^{8}$.

Situados en este contexto teórico, en este trabajo me propongo analizar los desarrollos teóricos de lo que se dio en llamar: "originalismo de intenciones originales" -también "viejo originalismo"-. Vale aclarar que aquí se examinarán las principales contribuciones teóricas contemporáneas al originalismo de intenciones originales que se gestaron en el ámbito estadounidense y cuyo origen se remonta a principios y mediados de los años ochenta9 ${ }^{9}$ Lo último de ningún modo implica negar la existencia de desarrollos teóricos en trabajos doctrinarios anteriores a esa época ${ }^{10}$ e,

4 Solum (2008) 6-8. Del mismo autor (2011) 33-35.

5 Solum (2008) 11. Los desacuerdos actualmente se profundizan a la hora de brindar una justificación normativa del originalismo; esto es, al momento de ofrecer las razones por las cuales los intérpretes deberían aplicar la metodología interpretativa que propugnan las versiones más recientes del originalismo, Whittington, Keith E. (2013). "Originalism: a Critical Introduction”. Fordham Law Review, vol. 82, pp. 375-409, pp. 394-400.

6 Solum (2011) 17. Alexander, Larry (2011). "Simple-Minded Originalism", (ed.) G. Huscroft and B. W. Miller, en The Challenge of Originalism: Theories of Constitutional Interpretation, New York: Cambridge University Press, pp. 87-98, p. 88.

7 Bork, Robert H. (1986). "The Original Intent and the Constitution", Humanities, vol. 7, $\mathrm{n}^{\circ} 1$, pp. 22-28, p. 26. Alexander, Larry (2013). "Originalism, The Why and The What", Fordham Law Review, vol. 82, pp. 539-544, pp. 540-541.

$8 \quad$ BORK (1986) 26.

9 Solum (2011) 16.

10 En rigor, podría rastrearse antecedentes teóricos del originalismo de intenciones originales en trabajos de principios del S. XX como, por ejemplo, Machen, Arthur W. Jr. (19001901): “The Elasticity of the Constitution". Harvard Law Review, vol. 14, n 3, pp. 200 
incluso, en la jurisprudencia de la Corte Suprema de los Estados Unidos de América ${ }^{11}$.

He optado por analizar los trabajos de autores de los años ochenta porque en ese tiempo el originalismo -en su versión intencionalista- comienza a presentarse como un movimiento teórico y práctico que pretende formular un paradigma interpretativo alternativo con la pretensión de guiar las prácticas interpretativas judiciales ${ }^{12}$. En tal sentido, como afirman Whittington y Goldford, el originalismo de intenciones originales pretendía ofrecer un marco alternativo -tanto teórico y práctico- a los controvertidos precedentes judiciales que dictó la Corte de Warren y sus herederos en materia de derechos civiles ${ }^{13}$. Más en concreto, esta última corriente de la teoría originalista se centró principalmente en dos cuestiones: la primera consiste en proponer un método interpretativo que permitiría garantizar la autorrestricción del Poder Judicial. Es decir, el originalismo de intenciones originales solía presentarse como un medio idóneo para limitar la discrecionalidad judicial en lo que hace a la interpretación de la Constitución ${ }^{14}$. En segundo término, la citada versión del originalismo pretendía otorgarle una preeminencia a las decisiones de las mayorías legislativas por sobre las interpretaciones del texto constitucional que pudieran desplegar los jueces. En síntesis, a partir de los años ochenta, el originalismo intencionalista aspira a consolidarse como un paradigma interpretativo alternativo capaz de enfrentarse con los marcos teóricos en los que se apoyan las controvertidas sentencias de la Corte de Warren, particularmente en materia de "derechos civiles" - civil rights, en la terminología constitucional estadounidense-.

216. Del mismo autor, (1900-1901): “The Elasticity of the Constitution II". Harvard Law Review, vol. 14, $\mathrm{n}^{\circ}$ 4, pp. 273-285. En el contexto de la interpretación legal, dentro de la tradición anglosajona, la remisión a las intenciones originales se puede remontar incluso hasta la obra de Blackstone quien mantenía que: "The best way to interpret the will of legislator is by exploring his intentions at the time the law was made." Blackstone, William (1765-1769): Commentaries of the Law of England, 808 pp., p. 15 [Se cita por la edición de WM. Hardcastle Browne, St. Paul: West Publishng Co., 1897]. Aún más, siempre en referencia a la interpretación de la ley positiva, también existen apelaciones a las intenciones de legislador como fundamento de la interpretación jurídica en los textos del Aquinate (S. XIII). Tomás de Aquino, Suma Teológica, I-II, q. 96, a. 6. [Se cita por la edición dirigida por los Regentes de Estudios de las Provincias Dominicanas en España, Madrid: B. A. C., 1998.]

11 Por ejemplo, “Holden v. Hardy" (1898): 169 U. S. 366, 390.

12 Más específicamente, "Although the originalism debate brewed quietly in academic and intellectual circles throughout the 1970 s, the general public's awareness of it was stimulated by the determined and single-minded jurisprudential agenda of the Reagan administration during the 1980s". Goldford, Dennis J. (2005). The American Constitution and the Debate over Originalism, New York: Cambridge University Press, 305 pp., p. 1.

13 Goldford (2005) 1. Whittington, Keith E. (2004). "The New Originalism”. The Georgetown Journal of Law \& Public Policy, vol. 2, pp. 599-613, p. 601.

$14 \quad$ Whittington (2004) 602. 
Así, la bibliografía que se estudiará proviene exclusivamente del mundo anglosajón - particularmente de los Estados Unidos de Américay, más específicamente, me focalizaré sobre las fuentes del originalismo de intenciones originales. Es decir, centraré las discusiones bibliográficas sobre los textos de los autores que defienden el originalismo de intenciones originales. Esta elección se justifica en dos razones principales. La primera es que el debate sobre la interpretación originalista de la Constitución ha tenido -y sigue teniendo- una incomparable difusión, extensión y profundidad en el constitucionalismo estadounidense contemporáneo. En segundo lugar, se puede advertir que prácticamente no existen trabajos monográficos en lengua castellana relativamente actualizados que discutan focalmente los principales desarrollos teóricos estadounidenses sobre la "familia de teorías" originalistas -en general-, ni del originalismo de intenciones originales -en particular- ${ }^{15}$. Asimismo, incluso en la literatura jurídica norteamericana apenas se ha ido más allá de las discusiones centradas en el concepto de autoridad y Constitución que presupone el originalismo de cuño intencionalista ${ }^{16}$.

15 Excepcionalmente, aunque se trata de una monografía que ha quedado desactualizada, LorA Del Toro, Pablo (1998). La interpretación originalista de la Constitución. Una aproximación desde la filosofía del derecho, Madrid: C.E.P.C., 343 pp. Vale aclarar que el citado autor no realizó investigaciones tendientes a examinar los avances y desarrollos de las teorías originalistas posteriores a la citada monografía. En cambio, se dedicó a investigar sobre temas de "ética aplicada", como "derechos de los animales" y "bioética". Lo último ha sido confirmado por el Prof. Lora del Toro en un intercambio de correos electrónicos con el autor de este trabajo. [Comunicación personal: 06-IV-2014]. En la doctrina hispanoparlante existen algunas otras pocas referencias conceptuales a la interpretación originalista de la Constitución en algunos trabajos panorámicos sobre temas de interpretación constitucional. En ese orden de ideas, cabría mencionar a Sagüés quien ha sostenido que toda interpretación constitucional presupone un determinado concepto de Constitución. En el caso del originalismo, según el citado autor argentino, este presupone una "Constitución-estatua" que implicaría un " $(. .$.$) cuerpo rígido, inmutable e incorrupto, compuesto por reglas también ya diseñadas$ que cabe lealmente cumplir. Y esa lealtad significa -básicamente- respetar la letra y el espíritu del constituyente histórico. La doctrina de la "Constitución-Estatua» se vincula con la idea de la "Constitución-testamento": un documento (ley fundamental) que fija las ideas y las órdenes del constituyente histórico, y que debe ser obedecido y realizado de modo que su ejecución cumpla exactamente con sus intenciones. El intérprete de la Constitución pasa aquí a ser el «albacea» de ella". SAGüÉs, Néstor Pedro (2006). La interpretación judicial de la Constitución, 2da edición, Buenos Aires: Abeledo-Perrot, 240 pp., p. 22.

16 Los estudios teóricos sobre el originalismo de intenciones originales, como señala Kavanagh, suelen centrarse casi exclusievamente en discutir o defender un determinado concepto de autoridad de la Constitución que presupone esa versión del originalismo. En efecto, la mayoría del los autores prestan escasa atención a las cuestiones metodológicas o a los presupuestos semánticos sobre los cuales se apoya la mencionada teoría interpretativa. KavanAGH, Aileen (2002). "Orginal Intention, Enacted Text, and Constitutional Interpretation", American Journal of Jurisprudence, vol. 47, pp. 255-298, p. 294. En contraste a lo que señala Kavanagh, en el ámbito latinoamericano cabe destacar un trabajo muy interesante que parte de las críticas al originalismo -en general, no solo del originalismo estadounidense- para luego proponer una serie de estándares interpretativos que justificarían el uso apropiado de una técnica interpretativa de corte originalista. Verdugo Ramírez, Sergio \& García G, 
Si bien el estudio de alguna de las versiones del originalismo estadounidense contemporáneo podría resultar un tema de sumo interés para la teoría constitucional norteamericana, a mi entender, una investigación sobre una de ellas en particular -el originalismo de intenciones originales- podría echar luz sobre la práctica constitucional argentina. En apoyo de esto último, vale recordar que las referencias a las "intenciones del constituyente" o a "las intenciones del legislador" no son infrecuentes en varias sentencias recientes de la Corte Suprema de Justicia de la Nación Argentina, incluso ante casos de suma relevancia institucional ${ }^{17}$. Más aún, el citado tribunal ha manifestado que la interpretación jurídica no se agota en el texto a interpretar sino que, además, debe dar pleno efecto a la intención del legislador ${ }^{18}$. Tales referencias a la jurisprudencia reciente del máximo tribunal argentino permiten sugerir que la remisión a las intenciones del constituyente $-\mathrm{o}$ del legislador- no representa simplemente un recuerdo del pasado. Antes bien, lo último exige discutir la consistencia metodológica del originalismo de intenciones originales. En otras palabras, el efectivo uso de directivas interpretativas originalistas en la jurisprudencia de la máxima instancia judicial argentina configura la ocasión propicia para estudiar críticamente los principales desarrollos teóricos del originalismo intencionalista.

Este trabajo se estructurará con el siguiente hilo argumental: una primera parte en la cual se enunciarán tres aseveraciones sintéticas sobre

José Francisco (2013). "El (mal) uso de la técnica originalista de interpretación. A propósito del Caso de Luciano Cruz-Coke", Revista de Derechos Fundamentales, n 10, pp. 137-156, p. 147. Beltrán de Felipe emplea una metodología de investigación similar a la de Verdugo Ramírez y García G., al punto que en la introducción de su trabajo sostiene: "(...) para reflejar la polémica entre Dworkin y Bork no he manejado, como fuentes originales, más que los artículos de este último, de modo que las teorías de Bork no quedan recogidas sino de forma indirecta”. Beltrán de Felipe, Miguel (1989). Originalismo e interpretación. Dworkin vs. Bork: una polémica constitucional, Madrid: Civitas, pp. 120, p. 24. En constraste, la metodología del presente trabajo es sustancialmente distinta a la empleada por los citados autores chilenos y a la del mencionado profesor español. En efecto, focalizaré mi interés teórico en examinar la consistencia metodológica del originalismo de intenciones originales a partir de la descripción de las tesis nucleares o centrales que mantienen las propias defensas teóricas de autores que intervinieron directamente en el debate originalista estadounidense -en su variante intencionalista $-\mathrm{y}$ no de las respuestas, observaciones o críticas a tales defensas.

17 La Corte Suprema de Justicia de la Nación Argentina ha justificado uno de sus fallos institucionales más relevantes de los últimos años con una apelación tanto al argumento de la "intención de legislador" como a las "intenciones del constituyente". Rizzo cl Poder Ejecutivo Nacional (2013), Corte Suprema de Justicia de la Nación 18 de junio 2013 (s/ Acción de Amparo c/ Poder Ejecutivo Nacional - Ley 26.855 - Medida Cautelar), consid. 16 y 22 del voto de la mayoría, respectivamente. Fisco Nacional Dirección General Impositiva cl Llámenos S.A. (2007), Corte Suprema de Justicia de la Nación 6 de noviembre de 2007. (s/ Recurso de Hecho), consid. 16. NSS S.A. c/ GCBA (2014), Corte Suprema de Justicia de la Nación 15 de julio de 2014 (s/ proceso de conocimiento), consid. 17.

18 Vale aclarar que el citado tribunal argentino no ha planteado distinciones significativas entre dar pleno efecto a las intenciones del legislador y dar pleno efecto a las intenciones del constituyente. Idem. 
las directivas interpretativas y los principales presupuestos semánticos del originalismo intencionalista. En la segunda parte de este artículo se esbozarán los lineamientos generales de dos posibles objeciones a esa teoría interpretativa originalista. Me permito dejar en claro que este trabajo de ningún modo tiene pretensiones de completa exhaustividad. El objetivo general es algo más modesto y se limita a introducir al lector hispanoparlante a los principales desarrollos teóricos contemporáneos del originalismo de intenciones originales desde las fuentes proveniente de la doctrina estadounidenses contemporánea y, a la vez, brindar una serie de observaciones críticas a esa teoría interpretativa originalista. En tal sentido, me permito aclarar que la valoración crítica que se realizará en este artículo, en buena medida, descansa en argumentos provenientes de la filosofía del derecho y la filosofía del lenguaje, como una buena parte de los desarrollos teóricos actuales en materia de interpretación constitucional. Sin embargo, el interés por estas últimas cuestiones filosóficas resulta estrictamente funcional al objetivo central o primordial de este trabajo, describir las tesis nucleares del originalismo de intenciones originales y señalar dos limitaciones metodológicas.

\section{1) PRIMERA TESIS: LA DISTINCIÓN ENTRE “INTENCIONES” Y “MOTIVOS"}

Una de las aseveraciones específicas sobre la que se apoya fundamentalmente el método interpretativo que se examina centralmente en el presente trabajo es la distinción entre "intenciones" y "motivos" 19 . Los últimos, como sostiene Michaels, refieren a los estados mentales de las personas que redactaron un texto ${ }^{20}$. De hecho, los miembros de una convención constituyente -originaria, ratificadora o reformadora- podrían haber concebido en su mente la consecución de determinados resultados particulares que impulsaron sus acciones dirigidas a redactar o aprobar las normas constitucionales puestas a su consideración. Por ejemplo, puede que un convencional constituyente haya propuesto una determinada moción con el objetivo de conseguir un beneficio personal de índole financiera ${ }^{21}$. Sin embargo, tal clase de estados mentales no configurarían

19 Michaels, Walter Benn (1989). "Intentionalism, Again", Cardozo Studies of Law \& Literature, vol. 1, pp. 89-96, pp. 89-90.

20 Michaels (1989) 90.

21 En la práctica legislativa argentina ha sido famoso el caso en el cual se discutía si el gobierno del presidente Fernando de la Rúa sobornó a senadores de los partidos opositores para que aprobaran la ley de reforma laboral. Finalmente, quedó firme la sentencia judicial en la que fueron absueltos todos los acusados. No obstante, suponiendo hipotéticamente que hubieran sido demostradas tales acusaciones, el interés de los legisladores por obtener las sumas de dinero prometidas no sería más que un "motivo" y no una "intención" de lo que los legisladores pretendieron significar con el texto normativo que aprobaron. Para una crónica 
más que motivos que de ningún modo podrían ser considerados como elementos relevantes o decisivos para "descubrir" 22 el significado lingüístico original de un concepto enunciado en el texto constitucional ${ }^{23}$.

En contraste, lo que sí resultaría relevante para el desarrollo de la interpretación jurídicas son las intenciones - no los motivos- de las personas que redactaron el texto constitucional. Estas últimas, como lo afirman Michaels, Kay y Alexander, remiten a lo que los autores efectivamente pretendieron significar con cada una de las formulaciones lingüísticas por medio de las cuales se enuncian las disposiciones constitucionales ${ }^{24}$. Más aún, el significado de una norma constitucional se reduciría a lo que los autores quisieron prescribir públicamente al momento de enunciar una norma constitucional a través de una determinada formulación lingüística incorporada al texto constitucional ${ }^{25}$.

\section{2) SEGUNDA TESIS: LOS TITULARES DE LA “INTENCIÓN ORIGINAL"}

Parafraseando a Dworkin, no solo es necesario determinar qué son las "intenciones originales" (original intent) sino que, ante todo, resulta imprescindible especificar cuáles son aquellas intenciones sobre las cuales se habrá de desarrollar la metodología interpretativa que propone el originalismo intencionalista ${ }^{26}$. Más concretamente, de quiénes son las

periodística del caso, H. Cappiello, "Absuelven a De la Rúa y al resto de los acusados en el caso de los sobornos”, La Nación [disponible en: www.lanacion.com.ar/1650534-despuesde-13-anos-la-justicia-absolvio-a-de-la-rua-en-el-escandalo-de-los-sobornos] [fecha de visita: 02 de octubre de 2014] pp. 2-6.

22 Nótese que fue absolutamente deliberado el uso de la palabra "descubrir" en el cuerpo de este trabajo. En efecto, resulta coherente con el originalismo no utilizar palabras como “determinación” o, incluso, "atribución”. Así, lo que he pretendido poner de relieve con el uso del vocablo "descubrir" es que la metodología interpretativa originalista postula que la interpretación se dirige estrictamente a dilucidar un hecho empírico de índole lingüístico que acaeció en un determinado momento del pasado. Entre quienes afirman esto último de modo directo o explícito, desde una defensa de una teoría originalista, Solum (2008) 51, nota 161 .

23 Michaels (1989) 90. Kavanagh (2002) 264. Maltz, Earl M. (1987) "The Failure of Attacks of Constitutional Originalism”, Constitutional Commentary, vol. 4, pp. 43-56, p. 49.

24 KaY, Richard S. (1988). "Adherence to The Original Intentions in Constitutional Adjudication: three Objections and Responses", Northwestern University Law Review, vol. 82, n 2, pp. 226-292, p. 231. Michaels (1989) 95. Alexander (2011) 94.

25 Alexander (2011) 87-88.

26 Dworkin, Ronald (1981) "The Forum of Principle”, New York University Law Review, vol. 56, pp. 469-518, pp. 475-476. En un sentido similar, Kay y Jaffa se preguntan de quiénes -de qué grupos-son las intenciones que resultan relevantes para la interpretación constitucional. Kay (1988) 245-251. JAFFA, Harry V. (1987) “What were the 'Original Intentions' of the Framers of the Constitution of the United States?", University of Puget Sound Law Review, vol. 10, pp. 351-448, p. 355. Para uno de los pocos estudios en lengua castellana que trata sobre el problema de los titulares de las intenciones originales, aunque se concentra exclusivamente en el debate entre Ronald Dworkin y Robert H. Bork, Beltrán de Felipe, 
intenciones relevantes para llevar a cabo la metodología interpretativa que propugna la versión del originalismo que se examina en este trabajo. Frente a esta última cuestión, las defensas del originalismo intencionalista han elaborado dos clases de respuestas.

(i) Algunos autores, como Maltz, Alexander y Berger, defienden la tesis de que el intérprete tendría el deber de llevar a cabo prácticas interpretativas dirigidas a determinar cuáles eran las intenciones originales de los Constituyentes al momento de sancionar el texto constitucional ${ }^{27}$. El originalismo de intenciones se apoyaría sobre una directiva interpretativa fundamental: el método para interpretar la Constitución supone la especificación de lo que públicamente pretendieron significar los constituyentes al enunciar cada una de las normas constitucionales ${ }^{28}$.

(ii) Existe una segunda variante del originalismo intencionalista que mantiene que las "intenciones originales" remiten al significado que los ratificadores -las personas que ratificaron el texto constitucional- pretendieron prescribir al momento de poner en vigencia el texto constitucional $^{29}$. Esta última variante, defendida por Lofgren, Rotunda y Maggs, presenta un particular interés cuando se trata de interpretar una Constitución que atravesó por un arduo proceso de ratificación. Más aún, si ese proceso ratificatorio tuvo lugar sin que las personas encargadas de ratificar el texto constitucional pudieran acceder a los registros taquigráficos de los debates que le antecedieron en la respectiva convención originaria. Esto último se puede advertir paradigmáticamente, como señala Lofgren, en el caso de la Constitución de Estados Unidos de América ${ }^{30}$.

Miguel (1989) Originalismo e interpretación. Dworikn vs. Bork, una polémica constitucional, Madrid: Civitas, pp. 120.

27 Maltz (1987) 43. Berger, Raoul (1988) “Originalist Theories of Constitutional Interpretation”, Cornell Law Review, vol. 73, pp. 350-358, pp. 350-351. Farber, Daniel. (1989). "The Originalism Debate: a Guide for the Perplexed", Ohio State Law Journal, vol. 49, pp. 1085-1106, p. 1086. Alexander (2011) 94. Del mismo autor (2013) 540.

28 Maltz (1987) 47. Más aún, se ha sostenido que "Interpreting the Constitution's general language according to our best understanding of the original intent of the Framers is the only way in which the Constitution can be law (...) No other method of constitutional adjudication can accomplish that". Bork (1986) 26 [El destacado me pertenece]. En un trabajo reciente, en un sentido prácticamente idéntico a esta última cita de Bork, Graglia, Lino A. (2011) "Originalism and the Constitution: Does Originalism Always Provide the Answer?, Harvard Journal of Law \& Public Policy, vol. 34, n 1, pp. 73-88, p. 86.

29 Lofgren, Charles A. (1988) “The Original Understanding of Original Intent?”, Constitutional Commentary, vol. 5, pp. 77-113, p. 79. Rotunda, Ronald D. (1988). "Original Intent, the View of the Framers, and the Role of Ratifiers", Vanderbilt Law Review, vol. 41, pp. 507-516, p. 512. MaGgs, Gregory E. (2009) "Which Original Meaning of the Constitution Matters to Justice Thomas?", New York University Journal of Law and Liberty, vol. 4, pp. 494-516, p. 497. 


\section{3) TERCERA TESIS: LA CENTRALIDAD DEL "SIGNIFICADO DEL HABLANTE"}

Desde el punto de una vista del concepto de "significado", el originalismo de intenciones originales se apoya en una distinción que ya se ha vuelto canónica en la filosofía del lenguaje contemporáneo. Me refiero a la diferencia entre "significado del hablante" -speakers meaning-y "significado de la oración" -sentence meaning_ 31 .

El "significado del hablante" se podría explicar a través de una respuesta a una típica pregunta que suele suscitar casi cualquier práctica comunicativa: “¿qué quiso decir el emisor $X$ al afirmar $Y$ ?” Lo dicho anteriormente, llevado al contexto de las prácticas comunicativas de índole constitucional, plantearía interrogantes tales como, por ejemplo, ¿qué fue lo que quisieron significar los constituyentes argentinos al sancionar la norma constitucional que establece: "Las declaraciones, derechos y garantías que enumera la Constitución, no serán entendidos como negación de otros derechos y garantías no enumerados; pero que nacen del principio de la soberanía del pueblo y de la forma republicana de gobierno"32?

En contraste, el "significado de las oraciones", siempre en el marco de una teoría originalista de la interpretación constitucional, mantiene que el contenido semántico de las normas que integran el texto constitucional -originario y reformado- se reduce al significado convencional que compartía un "usuario razonable" de la época en que ese texto fue puesto en vigencia. En efecto, la cuestión fundamental para descubrir o reconocer cuál era el significado de una cláusula particular del texto constitucional se podría reducir a interrogantes tales como, por ejemplo, “¿qué hubiera significado "penas crueles" para un usuario razonable del lenguaje de la época en que fue aprobada la octava enmienda de la Constitución de los Estados Unidos de América 33 ?

Así, en el campo del originalismo de intenciones originales, la idea de significado del hablante supone preguntarse centralmente qué fue lo que pretendieron prescribir los constituyentes al enunciar cada una de las normas que integran el texto constitucional ${ }^{34}$. Aún más, la noción de significado del hablante lo que propone distintivamente es que se le debe asignar preeminencia o prioridad a lo que pretendió comunicar el emisor

\footnotetext{
31 Solum (2008) 34. Para una referencia a defensas del originalismo de intenciones originales que citan a los textos de Grice: KaY (1988) 230-231 y también Alexander (2011) 88.

32 Art. 33 de la Constitución de la Nación Argentina. Para un estudio reciente sobre el significado original de la mencionada norma enunciada en el texto constitucional argentino, García-Mansilla, Manuel J. (2013). “¿Presunción de constitucionalidad o presunción de libertad? Un análisis desde el artículo 33 de la Constitución nacional”. Anales de la Academia Nacional de Ciencias Morales y Políticas, vol. 40, pp. 8-118.

33 Solum (2008) 51.

34 BERgER (1988) 351.
} 
- "significado del hablante"- por sobre lo que literalmente se transmitió -"significado de la oración"-.

Lo último resultaría especialmente relevante porque no siempre se guarda una estricta correspondencia entre lo que pretendió significar el autor del texto y lo que efectivamente fue comunicado por medio de los enunciados constitucionales. En definitiva, en caso de conflicto entre el significado convencional original de un concepto y aquello que fue pretendido por los constituyentes, el originalismo de intenciones originales sostiene que ha de prevalecer lo segundo por sobre lo primero.

\section{4) BALANCE SINTÉTICO}

La semántica sobre la cual se apoya el originalismo de intenciones originales, plantea que cuando una asamblea constituyente aprueba una Constitución existirían suficientes razones para afirmar que el significado de las normas constitucionales equivale a lo que los autores o ratificadores de esas normas pretendieron prescribir. A su vez, lo último depende de lo que la comunidad lingüística destinataria era capaz de reconocer como aquello que los autores quisieron prescribir a través de las formulaciones lingüísticas por las cuales fueron enunciadas cada una de las normas constitucionales ${ }^{35}$. Por consiguiente, los autores del texto constitucional se habrían servido del significado convencional de las palabras o frases al momento de enunciar cada una de las normas constitucionales ${ }^{36}$. Aún más, el contenido semántico de los enunciados constitucionales haría referencia exclusivamente a las convenciones lingüísticas que compartía una determinada comunidad de usuarios del lenguaje constitucional. En otros términos, lo que la asamblea constituyente pretendió significar resultaría inteligible para el resto de la comunidad lingüística en la medida en que tales normas remitan al significado convencional de las palabras y frases que integran el texto constitucional.

En consecuencia, el originalismo de intenciones originales descansaría en una concepción convencionalista del lenguaje constitucional y, por lo mismo, la mencionada versión del originalismo identifica el concepto de significado de las palabras que integran el texto constitucional con las convenciones semánticas de la época en que ese texto fue puesto en vigencia. Dicho de otro modo, la posibilidad de comprender el significado de las normas constitucionales depende de lo que convencionalmente se entendía cuando fue aprobada la Constitución.

\footnotetext{
35 Solum (2008) 39.

36 Kavanagh (2002) 272.
} 


\section{5) PRIMERA OBJECIÓN: LA INEVITABLE DISCRECIONALIDAD JUDICIAL}

La razón de mayor peso para hacer confluir el significado de las cláusulas constitucionales con las intenciones originales de los Constituyentes o ratificadores es una garantía de fijación y objetividad de la interpretación constitucional para evitar el peligro de que el texto constitucional se diluya en las preferencias morales o políticas de los jueces en los resultados de sus prácticas interpretativas ${ }^{37}$. No obstante, aún si se asumiera que la evidencia historiográfica $-v$. gr. diarios de debate de la Convención de Filadelfia de 1787- permitiera descubrir lo que pretendieron significar la comunidad de autores o ratificadores del texto constitucionales, ello solo podría llegar a resolver uno de los aspectos del problema de la discrecionalidad judicial. Más en concreto, si acaso fuera posible que esa metodología interpretativa posibilitara descubrir el significado lingüístico original de una determinada norma constitucional $-v$. gr. qué significaba "penas crueles" para la comunidad lingüística de los Constituyentes-, todavía quedaría irresuelto el problema de la desproporción entre la abstracción del lenguaje y la singularidad de los $\operatorname{casos}^{38}$.

En este orden de idas, el significado lingüístico original no excluiría completamente la posibilidad de la discrecionalidad porque aún persistiría la necesidad de que el juez decida, entre varias opciones posibles, si una sanción penal particular, aplicada en un caso concreto y real -premisas fácticas-, se adecua o no a lo que prescribe o exigen los enunciados constitucionales - premisas normativas-. En efecto, la conexión entre premisas fácticas y premisas normativas no se encuentra invariablemente determinada de antemano y suele requerir una decisión judicial que, al menos en algunas oportunidades, resulta inevitablemente discrecional.

Lo dicho hasta ahora también podría reforzarse con otro argumento que no tiene que ver exclusivamente con la conexión entre premisas normativas y premisas fácticas. En tal sentido, el intérprete jurídico no siempre es capaz de "descubrir" el significado lingüístico original sin adoptar una decisión en alguna medida discrecional frente a la resolución de un caso concreto. Lo último se enlaza con la nota específica que supone el concepto de "textura abierta" del lenguaje jurídico que bien resaltó Hart. Es decir, la posibilidad de que todas palabras del lenguaje -aún en sus instancias claras o paradigmáticas de aplicación-, en alguna oportunidad,

\footnotetext{
37 Maltz, Earl M. (1987b): "Foreword: The Appeal of Originalism”, Utah Law Review, vol. 4, pp. 773-807, p. 789. Más aún, Berger llegó a sostener que "Unless limited by the original intention, those words serve a crystal ball from which a judge, like a soothsayer, can draw for anything he wants. That flies in the face of the founder's distrust of judicial discretion". Berger (1988) 351.

38 Moore, Michael S. (1985). "A Natural Law Theory of Interpretation”, Southern California Law Review, vol. 58, pp. 277-398, p. 283.
} 
pueden llegar a tornarse vagas o imprecisas ${ }^{39}$. Dicho de otra manera, todas las palabras o frases del lenguaje jurídico son potencialmente vagas.

Por ejemplo, podría advertirse que la evidencia historiográfica revela que el concepto de "violencia doméstica" que recoge la Constitución estadounidense refiere a situaciones que la terminología constitucionalista argentina llamaría como "conmoción interior" ${ }^{40}$. Aún más, el juez podría interpretar en abstracto que el caso que se trae a su consideración se ubica claramente bajo el núcleo de significado de "violencia doméstica". Pero todo lo anterior no quita la posibilidad de que el juez al momento de atribuir ese significado a un hecho concreto, al menos en ciertos casos fronterizos o extremos, podría llegar a tener que apartarse del significado lingüístico original. Siguiendo con el ejemplo anterior, por más que al juez le resultase clarísimo el contenido semántico original de "violencia doméstica", incluso en caso que no hubiera dudas de que el caso traído a su consideración se adecua a ese significado lingüístico original, de todos modos podría suscitarse un caso concreto en que no cabría aplicar rígidamente ese significado. Lo último podría tener lugar, en un plano hipotético, si hubiera razones suficientes para creer que la intervención de una determinada fuerza de seguridad habría de generar más disturbios sociales que aquellos que pretende disipar y, a la vez, existieran otros medios idóneos para solucionar el caso.

Asimismo, la potencial vaguedad de todo el lenguaje jurídico -del cual el lenguaje constitucional no es la excepción sino, tal vez, su caso paradigmático-, se enfrenta con una característica propia de toda interpretación jurídica: la inevitable necesidad de conectar el descubrimiento del significado lingüístico original con los fines propios de las normas particulares que se interpretan y, al mismo tiempo, con las finalidades que justifican globalmente a la práctica social que denominamos "derecho" 41 . De hecho, el intérprete jurídico no "conoce por el gusto de conocer" sino que

39 Para un desarrollo del concepto de "textura abierta" en el marco de la teoría jurídica contemporánea, Hart, H. L. A. (1994) The Concept of Law, 2nd edition, Oxford: Clarendon Press, 315 pp., pp. 124-136. Del mismo autor, (1983) Essays in Jurisprudence and Philosophy, Oxford: Clarendon Press, 396 pp., p. 275. Para una posición crítica en relación a este último concepto en el planteamiento hartiano, Moore, Michael S. (1981) "The Semantics of Judging", Southern California Law Review, vol. 54, pp. 151-294, p. 201.

40 "(...) as used in the late eighteenth century "domestic Violence" referred to riots, rebellions, and other forms of harmful physical force within the territory of a political unit in context, within the boundaries of a state". Solum, Lawrence B. (2013) "Originalism and the Unwritten Constitution”, University of Illinois Law Review, vol. 5, pp. 1935-1984, p. 1941. Para un trabajo monográfico sobre el significado lingüístico original de "violencia doméstica" en la Constitución de los Estados Unidos de América, Stein, Mark S. (2009) "The Domestic Violence Clause in 'New Originalist' Theory", Hastings Constitutional Law Quarterly, vol. 37, n 1 , pp. 129-140.

41 Zambrano, Pilar (2009) La inevitable creatividad en la interpretación jurídica: una aproximación iusfilosófica a la tesis de la discrecionalidad, Méjico D.F.: UNAM, 86 pp., p. 60. 
conoce en orden a la realización de una acción humana particular, esto es, "conoce para obrar". Por consiguiente, quien participa en la práctica interpretativa del derecho asume implícita o explícitamente una finalidad que dota de sentido a una práctica interpretativa particular y, al mismo tiempo, a la práctica jurídica considerada globalmente.

Aquí no sería posible agotar exhaustivamente las cuestiones referidas a los fines del derecho. Ya es todo un lugar común en la filosofía del derecho contemporánea que existen muchos desacuerdos teóricos y prácticos sobre cuáles son tales fines y sus respectivas las limitaciones o posibilidades epistémicas. Con todo, precisamente la multiplicidad de opiniones sobre tales cuestiones refleja una de las principales razones que permiten afirmar que el intérprete jurídico no realiza un mero juicio silogístico en el cual se limita a reproducir o aplicar la formulación lingüística de una norma jurídica ante un caso particular ${ }^{42}$. Antes bien, el intérprete jurídico adiciona, crea o pone algo en la práctica interpretativa que no se apoya exclusivamente en la formulación lingüística por la cual se enuncian cada una de las normas jurídicas. Sin dudas que el intérprete no debiera desconocer completamente la relevancia de las formulaciones lingüísticas de las normas que está llamado a interpretar. El punto que se pretende destacar es que resulta inevitable asociar ese significado lingüístico original con la realización de determinadas finalidades -no siempre enunciadas en el texto normativo- que justifican tanto a la norma constitucional en particular como a la práctica interpretativa jurídica considerada de forma global o sistémica.

En definitiva, la diversidad de concepciones acerca de los fines del derecho $-\mathrm{y}$ de los fines que justifican a cada norma constitucional en particular-, y los variados grados de conocimiento que podrían operar en relación a tales finalidades, bastan para demostrar que cada intérprete no se limita a aplicar mecánica o silogísticamente al derecho. El intérprete jurídico, de un modo inevitable, termina agregando o creando algo que no estaba contenido en el significado lingüístico original.

\section{6) SEGUNDA OBJECIÓN: EL ARGUMENTO DEL REGRESO AL INFINITO}

Se suele afirmar que el argumento del regreso al infinito cumple una función significativa en muchas de las discusiones y problemas que han tenido lugar en la historia de la filosofía ${ }^{43}$. Dentro de los posibles usos

Zambrano (2009) 61.

Black, Oliver (1996). "Infinite Regress Arguments and Infinite Regresses", Acta Analytica, vol. 16/17, pp. 95-124, p. 95. Wieland, Jen Willem (2013). "Infinite Regress Arguments", Acta Analytica, vol. 28, pp. 95-109, p. 107. Más aún, se ha llegado a afirmar que el argumento del regreso al infinito ha sido utilizado tanto en la tradición filosófica occidental 
que podrían tener tales argumentos en relación a la filosofía del lenguaje, en su aplicación a la interpretación constitucional, se pueden advertir al menos dos variantes principales que, de diferentes modos, conducen a resultados que permiten evaluar la consistencia teórica de la metodología interpretativa originalista $y$, por ende, permiten sugerir, como lo hace Wieland, que "no es un error decir que los argumentos del regreso al infinito tienen su lugar apropiado entre las más poderosas herramienta a disposición del filósofo" 44 .

En primer término, se podría utilizar el argumento del regreso al infinito para refutar enunciados en virtud de que serían imposibles de ser cuantificados. En el campo de la interpretación constitucional, esta variante del argumento del regreso al infinito supone la imposibilidad de llevar a cabo la cuantificación de los elementos que podrían incluirse dentro del rango de convenciones semánticas que resultaría necesario interpretar para así determinar el significado de un enunciado normativo ${ }^{45}$. Con otras palabras, si no es posible cuantificar las convenciones semánticas necesarias, pues se incurriría en un regreso al infinito.

Esta primera variante del argumento que se examina en este epígrafe, se podría ilustrar con un análisis de una célebre sentencia de la Corte Suprema de Justicia de los Estados Unidos en la cual se determinó, entre otras cuestiones, el significado constitucional de "libertad" (liberty) que enuncia la decimocuarta enmienda de la Constitución de la citada nación norteamericana ${ }^{46}$. El citado precedente sostuvo que la libertad consiste en el derecho fundamental a que cada persona defina su propio concepto de "existencia, significado, universo y el misterio de la vida humana" 47.

Situados en el caso mencionado anteriormente, y desde una rígida visión convencionalista del lenguaje aplicada al originalismo de intencionales originales, cabría afirmar que el significado de "existencia", solo podría resultar inteligible a partir de una convención lingüística de la época en que fue aprobada o ratificada la Constitución que permitiera, a su vez, echar luz sobre lo que los constituyentes o ratificadores pretendieron pres-

como en la oriental, Gratton, Claude (2010) Infinite Regress Arguments, Dordrecht: Springer, 211 pp., p. xi. Cabe mencionar que el interés por el argumento del regreso al infinito se remota, al menos, hasta los textos del Estagirita. Me refiero específicamente a los Analíticos Posteriores, Libro 1, Capítulo 3, 72b5-25 [se cita por la edición bilingüe de W. D. Ross, Aristotle's Prior and Posterior Analytics, Oxford: Clarendon Press, 1957.]

44 Wieland (2013) 107. En un sentido prácticamente idéntico, Gratton (2010) xi.

45 Este variante del argumento del regreso al infinito se inspira en el siguiente trabajo, Black (1996) 95-124.

46 "Planned Parenthood of Southeastern Pennsylvania v. Casey": (1992) 505 U.S. 833, 851.

47 "At the heart of liberty is the right to define one's own concept of existence, of meaning, of the universe, and of the mystery of human life". "Planned Parenthood...", 505 U.S. 833, 852. El citado ejemplo se inspira en el siguiente trabajo, Zambrano, Pilar (2013). "What Do We Mean When We Speak of Rights? Fundamental Principles, Natural Law and Semantics in the Age of Pluralism", Pro manuscrito, Pamplona, passim, 23 pp., p. 9. 
cribir al enunciar la citada enmienda. En tal orden de ideas, la fijación que implica el originalismo de intenciones originales supondría la delimitación del rango de convenciones semánticas que habrá de integrar una determinada práctica interpretativa. Por consiguiente, una interpretación originalista de la Constitución, como la que propugna el originalismo de intenciones originales, estaría frente una secuencia limitada de convenciones semánticas - pasadas- que parecería posibilitar la interpretación del significado lingüístico original de una determinada norma constitucional.

No obstante, a pesar que existiría una cantidad determinada o finita de convenciones semánticas correspondientes a la época en que se aprobó o ratificó el texto constitucional, de ello no se derivaría inmediatamente que el intérprete constitucional actual fuera capaz de acceder al contenido de esas convenciones pasadas. En tal sentido, desde el punto de vista del originalismo intencionalista, la posibilidad cuantificar las convenciones a interpretar no parecería suficiente para evitar otra variante del argumento del regreso al infinito. Más específicamente, la tesis de la fijación que defiende el originalismo podría delimitar la cantidad de convenciones lingüísticas a interpretar pero no brindaría razones para interrumpir definitivamente la cadena de remisiones a convenciones lingüísticas de la época en que se aprobó o ratificó el texto constitucional.

Por ejemplo, si uno examinara fuentes historiográficas estadounidenses tales como los debates de Filadelfia parecería que uno podría concluir, tal como lo afirma Stein, que el concepto constitucional de "violencia doméstica" equivaldría a lo que actualmente se entiende como "conmoción interior" en el texto constitucional argentino ${ }^{48}$. Es decir, supondría graves situaciones de desorden público que dan lugar a un estado de sitio. Sin embargo, ¿en virtud de qué razón no habría que examinar otras fuentes historiográficas más para dilucidar lo que los constituyentes pretendieron significar con la expresión de "violencia doméstica"? ¿Cuándo se podría afirmar concluyente y definitivamente que no es necesario remitirse a una nueva convención semántica que recoge algún documento histórico para reconocer definitivamente qué es lo que los constituyentes pretendieron significar? ${ }^{49}$.

Solum (2013) 1941. Stein (2009) 129-140.

Beltrán de Felipe recoge un argumento similar, aunque no lo llama "regreso al infinito", cuando sostiene que el concepto de intenciones originales sobre el cual se estructura la versión del originalismo que se estudia en este trabajo presupone que tales intenciones originales son unívocas y no contradictorias con otras normas constitucionales. De hecho, si el significado al que remiten las intenciones originales no siempre es unívoco y remite a convenciones semánticas del pasado, no pareciera ser posible brindar un criterio para detener la cadena de remisiones. Así, siguiendo otro ejemplo típicamente utilizado por los críticos del originalismo intencionalista, no se podrían brindar criterios definitivos para decidir si ha de prevalecer el concepto de Constitución flexible de Jefferson o la Constitución rígida que Madison defendía en el capítulo 49 en el célebre El Federalista. El último ejemplo solo 
Desde una concepción convencionalista del lenguaje constitucional, como aquella sobre la cual descansa el originalismo de intenciones originales, no sería posible de ofrecer razones que justificarían detener tal encadenamiento de convenciones lingüísticas en algún eslabón definitivo. La semántica convencionalista sobre la cual descansa la inteligibilidad del originalismo de intencionalista supone un modo de concebir al lenguaje que bloquearía todo acceso a una realidad que no esté integrada por prácticas lingüísticas convencionales. Por consiguiente, el método interpretativo que propugna el originalismo de intenciones originales derivaría en una concepción del lenguaje estrictamente autorreferencial. Vale decir, un lenguaje que no hace más que discurrir sobre sí mismo, sin alcanzar algún aspecto de la realidad fuera de sí mismo ${ }^{50}$.

En definitiva, en virtud las coordenadas teóricas del convencionalismo semántico sobre el cual descansa el originalismo de intenciones, resultaría imposible detener el reenvío a convenciones lingüísticas de la época en que la Constitución fue aprobada y ratificada. Lo último se debe a que una semántica convencionalista es iterativa, es decir, conlleva necesariamente un pensamiento que se limita a reflexionar sobre cómo se piensa lo que se piensa. En efecto, un pensamiento iterativo resulta ser una operación intelectual pura y exclusivamente remitente que no resulta capaz de detenerse, de manera objetiva y definitiva, en algún momento. Por lo tanto, el convencionalismo semántico sobre el que reposa el originalismo de intenciones originales no puede, de ningún modo, superar la descripción o, mejor dicho, la constatación de una cadena indetenible de prácticas lingüísticas convencionales a las que refieren cada uno de los conceptos constitucionales.

\section{7) A MOdo DE CONCLUSIóN}

Las defensas teóricas del originalismo suelen respaldar, implícita o explícitamente, la sistematización de las tesis fundamentales sobre las cuales se asienta tradición originalista que ha formulado Lawrence Solum. Este último autor mantiene que existen dos afirmaciones centrales en las cuales convergen todos $-\mathrm{o}$ al menos casi todos- los autores que se autodenominan "originalistas": 1) la tesis de la fijación que mantiene que el contenido semántico de cualquier disposición constitucional fue fijado al momento en que el texto constitucional fue aprobado o ratificado; salvo que mediase una reforma o enmienda constitucional. 2) La tesis de la con-

pretende ilustrar como de la delimitación del material histórico a interpretar no se sigue la posibilidad de determinar objetivamente cuál es la opción que mejor se ajusta a las intenciones originales. Beltrán de Felipe (1989) 71. 
tribución por la cual se afirma que el significado original de la Constitución contribuye al contenido y desarrollo de las prácticas constitucionales actuales.

Con todo, incluso en las discusiones internas del originalismo, existen divergencias sobre las implicancias y consecuencias teóricas que se siguen de ambas tesis. Así, la diferencia específica que caracteriza al originalismo intencionalista es que el significado de las normas constitucionales remite a las intenciones originales de los constituyentes - framers- $\mathrm{o}$ también, en el caso estadounidense, a las intenciones de los ratificadores. Es decir, las personas encargadas de ratificar el texto constitucional en las asambleas o convenciones estaduales. La tesis de la contribución mantiene que el originalismo de intenciones originales es capaz de guiar a los intérpretes constitucionales actuales frente a la resolución de casos reales y concretos.

Respecto de las objeciones formuladas al originalismo de cuño intencionalista, vale destacar que este última teoría no logra evitar la discrecionalidad judicial. Lo dicho anteriormente se debe a que el juez inevitablemente no se limita a aplicar un puro silogismo al momento de evaluar si un caso particular se subsume -o no- al significado lingüístico original de una determinada norma constitucional. Además, tanto las variadas concepciones acerca de los finales globales del derecho y de las normas jurídicas en particular, como también los múltiples grados de conocimiento sobre tales fines, bastan para demostrar que la selección de la norma jurídica a la cual se subsume el caso concreto no es una operación exclusivamente mecánica o silogística. En otras palabras, el intérprete jurídico, al momento de subsumir un caso concreto al significado lingüístico original, no puede evitar terminar agregando o creando algo que no estaba meramente enunciado en el texto original de la Constitución.

Finalmente, la tesis de la fijación que caracteriza a las distintas teorías que integran la familia de teorías originalistas podría implicar que no existe una cantidad infinita de convenciones lingüísticas a las que remiten el significado de cada una de las normas constitucionales. Desde la versión del originalismo de intenciones originales, la fijación implicaría que no existe una cantidad ilimitada de convenciones lingüísticas pasadas. Sin embargo, el originalismo intencionalista no podría brindar razones para que el intérprete constitucional se detenga en alguno de los eslabones que integran la mencionada cadena de convenciones que describen el significado lingüístico original. Dicho de otro modo, el originalismo intencionalista podría suponer una delimitación bastante precisa de la cantidad de convenciones lingüísticas a interpretar pero no brindaría criterios objetivos para determinar en qué momento cabría detener, de modo definitivo, la cadena de remisiones a otras convenciones semánticas pasadas. 


\section{8) Bibliografía}

- Alexander, Larry (2011) "Simple-Minded Originalism", (ed.) G. Huscroft and B. W. Miller. The Challenge of Originalism: Theories of Constitutional Interpretation, New York: Cambridge University Press, pp. 87-98.

- -(2013) "Originalism, The Why and The What", Fordham Law Review, vol. 82), pp. 539-544.

- Aristóteles, Analiticos Posteriores, [se utilizó la edición bilingüe de W. D. Ross, Aristotle's Prior and Posterior Analytics, Oxford, Clarendon Press, 1957.]

- Barnett, Randy E. (2013) "The Gravitational Force of Originalism”, Fordham Law Review, vol. 82, pp. 411-432.

- Beltrán de Felipe, Miguel (1989). Originalismo e interpretación. Dworikn vs. Bork, una polémica constitucional, Madrid: Civitas, pp. 120.

- Berger, Raoul (1988) "Originalist Theories of Constitutional Interpretation”, Cornell Law Review, vol. 73, pp. 350-358.

- Black, Oliver (1996) "Infinite Regress Arguments and Infinite Regresses", Acta Analytica, vol. 16/17, pp. 95-124.

- Blackstone, William (1765-1769) Commentaries of the Law of England, 808 pp. [Se utilizó la edición de WM. Hardcastle Browne, St. Paul: West Publishng Co., 1897].

- Bork, Robert H. (1986) "The Original Intent and the Constitution", Humanities vol. 7, $\mathrm{n}^{\circ}$ 1, pp. 22-28.

- Dworkin, Ronald (1981) "The Forum of Principle", New York University Law Review, vol. 56, pp. 469-518.

- Farber, Daniel (1989) "The Originalism Debate: a Guide for the Perplexed", Ohio State Law Journal, vol. 49, pp. 1085-1106.

- García-Mansilla, Manuel J. (2013) "Presunción de constitucionalidad o presunción de libertad? Un análisis desde el artículo 33 de la Constitución nacional", Anales de la Academia Nacional de Ciencias Morales y Politicas, vol. 40, pp. 8-118.

- Goldford, Dennis J. (2005) The American Constitution and the Debate over Originalism, New York: Cambridge University Press, 305 pp.

- Graglia, Lino A. (2011). "Originalism and the Constitution: Does Originalism Always Provide the Answer?, Harvard Journal of Law \& Public Policy, vol. 34, $\mathrm{n}^{\circ}$ 1, p. 73-88.

- Gratton, Claude (2010) Infinite Regress Arguments, Dordrecht: Springer, $211 \mathrm{pp}$.

- Hart, H. L. A. (1983) Essays in Jurisprudence and Philosophy, Oxford: Clarendon Press, 396 pp. 
- $\quad$ (1994). The Concept of Law, $2^{\text {nd }}$ edition, Oxford: Clarendon Press, $315 \mathrm{pp}$.

- JAfFa, Harry V. (1987) "What were the 'Original Intentions' of the Framers of the Constitution of the United States?", University of Puget Sound Law Review, vol. 10, pp. 351-448.

- Kavanagh, Aileen (2002) "Orginal Intention, Enacted Text, and Constitutional Interpretation", American Journal of Jurisprudence, vol. 47, pp. 255-298.

- KaY, Richard S. (1988) "Adherence to The Original Intentions in Constitutional Adjudication: three Objections and Responses", Northwestern University Law Review, vol. 82, n 2, pp. 226-292;

- -(2009) "Original Intention and Public Meaning in Constitutional Interpretation", Northwestern University Law Review, vol. 103, $\mathrm{n}^{\circ} 2$, pp. 703-726.

- Llano, Alejandro (1984) Metafísica y lenguaje, Pamplona: EUNSA, 363 pp.

- Lofgren, Charles A. (1988) "The Original Understanding of Original Intent?", Constitutional Commentary, vol. 5, pp. 77-113.

- Lora Del Toro, Pablo (1998) La interpretación originalista de la Constitución. Una aproximación desde la filosofía del derecho, Madrid: C.E.P.C., 343 pp.

- Machen, Arthur W. Jr. (1900-1901) "The Elasticity of the Constitution", Harvard Law Review, vol. 14, n 3, pp. 200-216;

- - (1900-1901). "The Elasticity of the Constitution II", Harvard Law Review, vol. 14, n 4, pp. 273-285.

- Maggs, Gregory E. (2009) "Which Original Meaning of the Constitution Matters to Justice Thomas?", New York University Journal of Law and Liberty, vol. 4, pp. 494-516.

- Mattz, Earl M. (1987) "The Failure of Attacks of Constitutional Originalism", Constitutional Commentary, vol. 4, pp. 43-56.

- -(1987b) "Foreword: The Appeal of Originalism", Utah Law Review, vol. 4, pp. 773-807.

- Michaels, Walter Benn (1989) "Intentionalism, Again", Cardozo Studies of Law \& Literature, vol. 1, pp. 89-96.

- Moore, Michael S. (1981) "The Semantics of Judging", Southern California Law Review, vol. 54, pp. 151-294;

- -(1985) "A Natural Law Theory of Interpretation", Southern California Law Review, vol. 58, pp. 277-398.

- Rotunda, Ronald D. (1988) "Original Intent, the View of the Framers, and the Role of Ratifiers", Vanderbilt Law Review, vol. 41, pp. 507-516.

- SagüÉs, Néstor Pedro (2006) La interpretación judicial de la Constitución, $2^{\mathrm{da}}$ edición, Buenos Aires: Abeledo-Perrot, 240 pp. 
- Solum, Lawrence B. (2008) "Semantic Originalism". Illinois Public Law and Legal Theory Research Papers, Series No. 07-24. 176 pp. Disponible en: http://papers.ssrn.com/sol3/papers.cfm?abstract_ $\mathrm{id}=1120244$ [fecha de visita: 02 de octubre de 2014]

- -(2011) "What is Originalism?: The Evolution of Contemporary Originalist Theory", (ed.) G. Huscroft and B. W. Miller. The Challenge of Originalism: Theories of Constitutional Interpretation, New York, Cambridge University Press, pp. 12-41.

- - (2013) "Originalism and the Unwritten Constitution", University of Illinois Law Review, vol. 5, pp. 1935-1984.

- Stein, Mark S. (2009) "The Domestic Violence Clause in 'New Originalist' Theory", Hastings Constitutional Law Quarterly, vol. 37, $\mathrm{n}^{\circ} 1$, pp. 129-140.

- Tomás de Aquino, Suma Teológica, I-II. [se cita por la edición dirigida por los Regentes de Estudios de las Provincias Dominicanas en España, BAC, 1998]

- Verdugo Ramírez, Sergio \& García G, José Francisco (2013) "El (mal) uso de la técnica originalista de interpretación. A propósito del Caso de Luciano Cruz-Coke", Revista de Derechos Fundamentales, ${ }^{\circ}$ 10, pp. 137-156.

- Whittington, Keith E. (2004) "The New Originalism", The Georgetown Journal of Law \& Public Policy, vol. 2, pp. 599-613.

- -(2013). "Originalism: a Critical Introduction", Fordham Law Review, vol. 82, pp. 375-409.

- Wieland, Jen Willem (2013) "Infinite Regress Arguments", Acta Analytica, vol. 28, pp. 95-109.

- Zambrano, Pilar (2009) La inevitable creatividad en la interpretación juridica: una aproximación iusfilosófica a la tesis de la discrecionalidad Méjico D.F.: UNAM, 86 pp.

- -(2013). "What Do We Mean When We Speak of Rights? Fundamental Principles, Natural Law and Semantics in the Age of Pluralism", Pro manuscrito, Pamplona, $23 \mathrm{pp}$.

\section{JURISPRUDENCIA CITADA:}

1. Jurisprudencia estadounidense : Cabe aclarar que se cita por el nombre de las partes, el año en que fue dictada la sentencia, la fecha de esta última, el número de tomo, la referencia a los United States Reports (US)-cuya sola mención implica que se trata de la Corte Suprema de los Estados Unidos-, y la página en donde comienza el fallo. Este es el método con el cual suelen citar las sentencias del mencionado tribunal estadounidense. 
- "District of Columbia v. Heller" (2008): 26 de junio de 2008, 554 U.S.,570.

- "Holden v. Hardy", (1898): 28 de febrero de 1898, 169 U.S., 366.

- "Planned Parenthood of Southeastern Pennsylvania v. Casey", (1992): 29 de junio de 1992, 505 U.S. ,833.

2. Jurisprudencia argentina

- Fisco Nacional Dirección General Impositiva c/ Llámenos S.A. (2007), Corte Suprema de Justicia de la Nación 6 de noviembre de 2007. (s/ Recurso de Hecho), pp. 1-17. [Fecha de consulta: 1212-2014] [en línea: www.csjn.gov.ar/jurisp/jsp/fallos.do?usecase $=\operatorname{mos}$ trarDocumento\&falloId $=2577]$.

- Rizzo cl Poder Ejecutivo Nacional (2013), Corte Suprema de Justicia de la Nación 18 de junio 2013 ('s/ Acción de Amparo c/ Poder Ejecutivo Nacional - Ley 26.855 - Medida Cautelar), pp. 1-67. Disponible [Fecha de consulta: 12-12-2014] [en línea: http:// servicios.csjn.gov.ar/confal/ConsultaCompletaFallos.do?method=ver Documentos\&id=702685].

- NSS S.A. cl GCBA (2014), Corte Suprema de Justicia de la NACión 15 de julio de 2014 (s/ proceso de conocimiento), pp. 1-15. [Fecha de consulta: 12-12-2014] [en línea: http://servicios.csjn.gov. $\mathrm{ar} / \mathrm{confal} /$ ConsultaCompletaFallos.do? $\mathrm{method}=\mathrm{verDocumentos} \&$ $\mathrm{id}=712961]$. 